\title{
Extending and Improvising Service Discovery in MANET-relevant work and future proposal
}

\author{
Sumana Sinha \\ Information Science and Engineering \\ PESIT South Campus Bangalore \\ Bangalore, India \\ sumanasinha@pes.edu
}

\author{
Snehanshu Saha \\ Computer Science and Engineering \\ PESIT South Campus Bangalore \\ Bangalore, India \\ snehanshusaha@pes.edu
}

\begin{abstract}
Web services are the key technologies for the web applications developed using Service Oriented Architecture (SOA). Many outsourced web services can be combined to provide value added services to the users. There are many challenges involved in its implementations. One of the essential challenges is service discovery which involves finding a set of suitable web service in dynamic environment like MANET. When a large number of functionally equivalent services are discovered, it is difficult for a mobile node to discover a service. There are various techniques for service discovery like ranking and matchmaking the web services based on their Quality of Service (QoS). This paper gives an idea on selecting services based on various parameters of quality such as response time, availability, accessibility etc[15]. In this paper we propose a future scheme on service discovery considering the quality based on non-functional properties (NFPs) [7].
\end{abstract}

Keywords- Functional properties (NFPs), Service Oriented Architecture SOA, Quality of services (QoS), Web Service, MANET, Binomial Probability, Normal Distribution.

\section{INTRODUCTION}

The mobile Ad-hoc network (MANET) represents a complex distributed system that comprise wireless mobile nodes that can freely and dynamically self-organize into arbitrary and temporary, "ad-hoc" network topologies, allowing devices to seamlessly internetwork in areas with no pre-existing communication infrastructure \& its widespread usage of advanced multimedia capabilities and web services has given raised to its popularity. As Mobile nodes are known for its limited resource, necessitating it to cooperate among them for performing tasks that cannot be done alone. This cooperation is in the form of services that are offered by other devices in the network. To get benefit from the services offered by other devices, they have to be discovered [1].As it is known that the process of service is a method in which services can be regarded as any hard or software resource, which can be used by other clients and this service discovery is the process of locating services in a network.

The following are the basic methods used to discover and maintain service data:

- service providers flood the network with service advertisements;

- clients flood the network with discovery messages;
- $\quad$ nodes which are mobile and heterogeneous in nature caches the service advertisements;

- Node overhears in the network traffic and cache the interesting data.

So, due to the widespread adoption of SOA, which results in a rising number of clients and servers, QoS is becoming a key feature in Web services discovery. End-to-end quality management of Web services is a key issue that is highly related to the changing and dynamic environment like MANET [1]. Most of the research works on QoS support in Web services in MANET have focused on the enumeration of QoS requirements and mechanisms for QoS management. Also, some research efforts have addressed the issues of QoS manageability. The success of QoS support for Web services in these environments largely depends on the scalability of the proposed mechanisms for QoS management.

This paper proposes a framework where mobile Ad-hoc nodes discover services in distributed registries [2] and selecting them based NFP's which are often called qualities of a system by applying binomial distribution and thus maintaining availability and scalability of the service provided.

\section{RELATED WORK}

There has been significant research in web service discovery in MANET to cope up with QoS management. Qusay H. Mahmoud [5] proposed a solution based on QoS metrics that are not necessarily supplied by service providers (i.e. response time, availability, reliability, penalty rate among others) which are computed in an autonomous manner. They introduced the Web Service Relevancy Function (WsRF) used for measuring the relevancy ranking of a particular Web service based on client's preferences and QoS metrics. They proved that the use of non-functional properties for Web services significantly improves the probability of service discovered. Balfagih and Hassan [4] examined the quality of service (QoS) of SOA and web services, and classified them from the perspectives of the developer, provider, and consumer. Their list of QoS from the perspective of the consumer includes response time, availability, reliability, security, usability, and robustness. They defined the first three QoS and proposed corresponding metrics. For security, they mentioned a few sub-factors including confidentiality, integrity, authentication, and availability. They defined usability but again they did not propose any metrics; they simply mentioned sub-factors 
including understanding ability and configurability. They defined compos ability but they do not provide metrics. They proposed the following sub-factors that facilitate ability to compose, conformability, availability, discoverability, and the loosely coupled nature of the web services. These subfactors are the ones proposed in their model for the developer's and provider's perspectives. This paper provides a good literature review and QoS classification. However, there is no validation methodology or justification of the selected or eliminated QoS. Formal definitions and metrics are provided only for a few QoS. Elarbi Badidi and Larbi Esmahi [16] proposed a framework based on a federation of QoS Brokers that are in charge of mediating between service requestors and service providers, and carrying out various QoS management operations, where they describe an auction-based ranking algorithm of functionally equivalent services, which ranks services according to their ability to fulfil the service requestor QoS requirements. The brokers are also in charge of handling appropriately service requests from mobile users equipped with various handheld devices.

\section{OUR WORK}

The objective of our research work is to enable Mobile Adhoc nodes to discover services in distributed based on NFP's, where the nodes in the MANET can be a provider or user of Web Services and all communication including the one between the user and the provider is performed using SOAP [11] and the UDDI Registry is a mobile node which always keeps a backup for QoS calculation and update information of the web services which can be called as the extended UDDI Registry [2].

This framework enables Mobile Ad-hoc nodes to discover services in a distributed environment [2,6] based on NFP's, and to provide a QoS model for the specification of Web services in nodes where selection of services is based on parameters like throughput, response-time, cost of service etc. Thus, the model meets the following requirements:

- Providing the best available services present in the nodes in MANET using proper service discovery protocol (e.g. UDDI [7] or DAML [9]) and maintaining a backup UDDI Registry which is not globally accessible, where the services are invoked using SOAP. SOAP is a vital component Web Service which provides a standardized mechanism for heterogeneous information system. In order to send SOAP message, SOAP over UDP is used [8].

- Enabling the backup registry to calculate QoS for example the throughput of a service (TH) being invoked per unit time and providing it to the UDDI Registry and also keeping all the information related to the web services.

- Agent node's search messages on behalf of user depending on the QoS value, and these Agents are also capable of connecting with neighbor agent.

- This protocol allows calculating the probability of finding a service in the UDDI Registry which is done by the agent node based on the $\mathrm{TH}$ value obtained from the UDDI Registry. Thus, based on which the agent gives information of the service availability to the user with QoS. So Agent can act on behalf of the user and the provider.

- The non-functional parameters can be defined with help of XML based language if it is a web service (WSDL) [11] which are updated time to time by the UDDI backup.

To meet the above requirements, we propose a proper matchmaking algorithm to discover the services for the requester and thus ensuring the availability \& scalability of the service provided

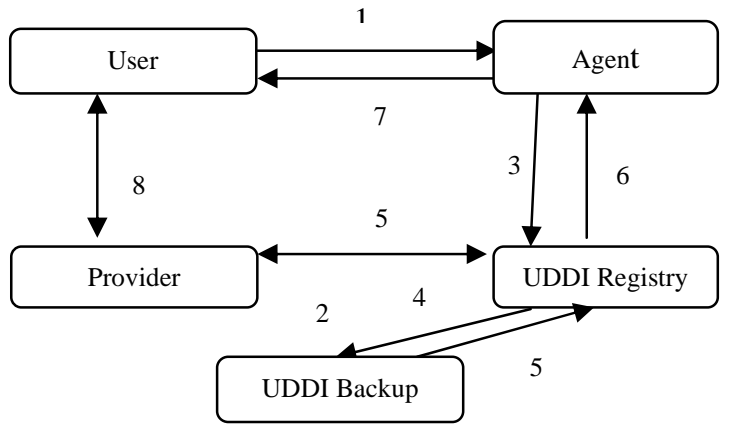

1. Web Service Request

2. Provider publishes in UDDI Registry

3. Agent looks for service in UDDI registry

4. UDDI Registry sends message to UDDI Backup for updating

5. UDDI Backup sends the calculated QoS of Web Service to the UDDI Registry and updates the related information.

6. UDDI Registry sends the information to Agent \& Provider.

7. Agent send presence of service message

8. User goes the service to the provider \& Provider provides

Fig 1.Selection of service in this protocol

Algorithm:

1. Agent sends a request on behalf of user in the UDDI Registry where the registry accumulates similar type of services and filter.

2. Let $n$ is the number of filtered services and $X$ is the number of services the agent is looking on behalf of the user.

- Calculate the throughput (TH) by calculating number of times a service is invoked per unit time in the UDDI Backup, where the TH corresponds to parameter $p$ depending on which the $q$ value can also be calculated.

Therefore, the probability of Agent discovering a Web Service correctly by binomial distribution [12] is:

$$
P(X=x)=p(\mathrm{x})=C_{x}^{n} \cdot p^{x} \cdot q^{(1-p)}
$$

Where $x=01,2,3 . . n ; q=1-p$ 
- Calculate the response-time (RT) as RT=1/TH and hence if RT is high the cost of service (CS).

3. The Agent finds the Web Service.

- Checks if the service found is from another Agent or not.

- If yes, then calculate the number of hops, which is the distance between the User and the Provider [10].

4. User goes for the desired Web Service from the Provider.

\section{A Toy Example}

For instance the user sends a message to an Agent to look on behalf of it in the UDDI Registry, where following are the assumptions considered:

\section{parameter $p=35 \%$}

$n$ (Similar type of Web Services) $=6$,

$x$ (Web Services the agent is looking) $=3$ with

Thus the probability that a services found by the agent is

$$
\left.P(X=3)=? C_{3} .0 .35\right)^{3} \cdot(0.25)^{7} \approx 0.25
$$

And if the probability that the agent never finds the service is:

$$
P(0)=q^{7} \approx 0.05
$$

Thus, the probability that the agent finds a target at least once is $0.95 \%$.

The Binomial distribution is used for less number, but for large $n$ and neither $p$ nor $q$ is very small considering the standard Binomial variate:

$$
Z=\frac{X-n p}{\sqrt{n p q}} ; X=0,1,2 \ldots \ldots, n
$$

$$
\text { When } X=0, Z=\frac{-n p}{\sqrt{n p q}}=-\sqrt{n p / q} \text {; }
$$

And

$$
X=n, Z=\sqrt{\frac{n p}{p}}
$$

Thus in the limit as $n \rightarrow \infty$ takes the values from $-\infty$ to $\infty$. Hence the distribution of $X$ will be continuous over the range $-\infty$ to $\infty$, and the limiting form of (1) under the above two condition is given by normal distribution with mean $\mu$ and standard deviation $\sigma$ is given by:

Theorem 1: if $x$ is number of services agent is looking, then the maximum probability occurring at the point $x=\mu$ is:

$$
[p(x)]_{\max }=\frac{1}{\sigma \sqrt{2 \pi}}
$$

Sketch of Proof: Since,

$$
f(\mathrm{x})=\frac{1}{\sigma \sqrt{(2 \pi)}} e^{-(\mathrm{x}-\mu)^{2} / 2 \sigma^{2}},-\infty<x<\infty
$$

Where, $\mu=n p$ and $\sigma=\sqrt{n p q}$.

So, in terms of binomial distribution, we determine that the success of service selection based on NFP's and above we have considered one of its i.e. throughput which is based on the number of time it is invoked is very high and thus ensuring the availability and reliability of a service being selected and served to the user and also to make it scalable we can use normal distribution for large number of $n$, where maximum occurrence can be found by (4).

\section{SIMULATION}

To simulate the framework, Franson Bluetools and PockectSOAP toolkit is used. Franson Bluetools is an SDK for the fast and easy development of Bluetooth enabled .Net applications and PocketSOAP is a SOAP client toolkit for windows, and a Bluetooth environment using 6 mobile devices which support the Bluetooth stack and a note book with Pentium processor and $1 \mathrm{G}$ memory and the setup have 2 mobile device as Web Service provider, 1 device as Web Service user and the other two as UDDI Registry and UDDI Backup.

\section{CONCLUSION}

The advantage of this Service Discovery architecture helps in selecting best available service during service discovery process based on a set QoS. The application of binomial and limiting form of it i.e. normal distribution on non-functional properties significantly improves the probability of having relevant output results. Incorporating QoS parameters as part of the search criteria and its ability to distinguish services from one another during the discovery process is effective. The ability to discriminate appropriate services relies on the client's ability to identify appropriate QoS parameters such as integrity, reputation, reliability, to name a

\section{REFERENCES}

[1] Deployment of SOA in MANET: a Research roadmap By Prasenjit Choudhury, Anirban Sarkar, Narayan Debnath. 2011 IEEE conference.

[2] Extended Web Services Framework to meet Non Functional Requirements by Abad Ali Shah, Zafar U.Singhera, University of Engineering and Technology, Lahore, Pakistan.

[3] Survey of Service Discovery Protocols in Mobile Ad Hoc Networks Technical Report 4/06 by Adnan Noor Mian, Roberto Beraldi, Roberto Baldoni California, in IEEE 2003.

[4] Z. Balfagih, M.F. Hassan, and M.F. "Quality Model for the web services from multi-stakeholders' perspective”, 
in Information Management and Engineering (ICIME'09), pp. 287-291, Kuala Lumpur.

[5] QoS-based Discovery and Ranking of Web Services by Eyhab Al-Masri and Qusay H. Mahmoud in Computer Communications and Networks, 2007. ICCCN 2007.

[6] Christopher N. Ververidis and George C. Polyzos, Member, IEEE Service Discovery for Mobile Ad (used)Hoc Networks: A Survey of Issues and Techniques.

[7] A Model for Web Services Discovery with QoS by Shuping Ran CSIRO Mathematical and Information Sciences, published in ACM SIGecom Exchanges in 2003.

[8] Cheng Zang, Zi-yu Chen, 2009 IEEE, A Novel Framework for Web Service in MAMET.

[9] V. Issarny, D. Sacchetti, F. Tartanoglu, Developing ambient intelligence systems: A solution based on Web Services. Journal of Automated Software Engineering, 2004.

[10] C. Makris, Y. Panagis, E. Sakkopoulos \& A. Tsakalidis
(2006): Efficient and adaptive discovery techniques Of web services handling large data sets. Journal of Systems and Software 79(4), pp. 480-495.

[11] WSDL, UDDI, SOAP, rest: power point presentation by Matthew J. Dovey Oxford University.

[12] Advanced engineering mathematics by H.K Dass.

[13] Service discovery in MANET via biased random walks by Roberto Beraldi in 2007.

[14] Non-functional Parameters as First Class Citizens in Service Description and Matchmaking - An Integrated Approach by Mohamed Hamdy, Birgitta Kaonig-Ries, and Ulrich Kauster, Institute of Computer Science.

[15] Mitigating Reply Implosions in Query-Based Service Discovery Protocols for Mobile Wireless Ad Hoc Networks by Antonio Tadeu A. Gomes, Artur Ziviani, Luciana S. Lima.

[16] Scalable Framework for Policy-based QoS Management in SOA Environment by Elarbi Badidi 